\title{
Prevalência de sinusopatias maxilares por meio de tomografia computadorizada de feixe cônico
}

\author{
- Cinthia Palmira Barbosa Departamento de Radiologia Dentomaxilofacial, Faculdade de Medicina e Odontologia \\ São Leopoldo Mandic, Campinas, SP, Brasil • Milena Bortolotto Felippe Silva Departamento de Radiologia \\ Dentomaxilofacial, Faculdade de Medicina e Odontologia São Leopoldo Mandic, Campinas, SP, Brasil • Juliane \\ Pirágine Araujo Departamento de Estomatologia, Faculdade de Odontologia, Universidade de São Paulo, São Paulo, \\ SP, Brasil - Ricardo Raitz Departamento de Radiologia Dentomaxilofacial, Faculdade de Medicina e Odontologia \\ São Leopoldo Mandic, Campinas, SP, Brasil
}

RESUMO || O objetivo deste trabalho foi avaliar a prevalência de sinusopatias nos seios maxilares (SM) por meio de tomografias computadorizadas de feixe cônico (TCFC) da maxila em pacientes selecionados de acordo com gênero e idade para o planejamento de implantes dentários. As 226 imagens tomográficas selecionadas foram analisadas independentemente por três cirurgiões dentistas (CD) especialistas com o intuito de verificar a presença de sinusopatias. As anormalidades encontradas foram: velamento sinusal, espessamento mucoso, pseudocisto antral, pólipos, e pneumatização. Das 226 TCFC analisadas, encontramos a presença de sinusopatias em 156 (69\%), sob a forma de velamento sinusal em 69 (30,5\%), espessamento mucoso em 63 (27,9\%), pneumatização em 57 (25,2\%), pseudocisto antral em 29 (12,8\%) e pólipos em seis (2,7\%), podendo ocorrer isolada ou simultaneamente. Quando observamos a ocorrência simultânea de duas ou mais sinusopatias, estas estiveram presentes em $56(24,8 \%)$ das TCFC observadas na amostra. O espessamento mucoso e pneumatização foram as mais frequentes, tendo ocorrido em $8,4 \%$ da amostra. Quanto ao gênero, não houve diferença entre os gêneros masculino e feminino no que diz respeito à prevalência das sinusopatias nos SM. Houve diferença entre faixas etárias apenas para pólipo, sendo que indivíduos com idade acima de 61 anos apresentaram maior taxa de prevalência. Nosso estudo mostra a importância da TCFC para implantes na maxila posterior em razão da alta prevalência de sinusopatias encontradas em pacientes assintomáticos.

DESCRITORES | Tomografia Computadorizada de Feixe Cônico; Seio Maxilar; Diagnóstico por Imagem.

ABSTRACT | Prevalence of maxillary sinusopathies using cone beam computed tomography - This study aimed to evaluate the prevalence of sinusitis in MS through computerized tomography of cone beam (CTCB) of the maxilla in patients selected for the planning of dental implant according to gender and age. All $226 \mathrm{CT}$ images were analyzed and visualized independently by three specialized surgeon dentists (SD) to verify the presence of sinusitis. Those changes of normality were classified as: sinus opacification, mucosal thickening, antral pseudocyst, polyps, and pneumatization. We found the presence of sinus diseases in 156 (69\%) out of the 226 CTCB analyzed, under the form of sinus opacification in 69 (30.5\%), mucosal thickening in 63 (27.9\%), pneumatization in 57 (25.2\%), antral pseudocyst in 29 (12.8\%) and polyps in six (2.7\%), which occurred in an isolated way or simultaneously. When we observe the simultaneous association of two or more sinus, we found them in $56(24.8 \%)$ out of all the CTCB observed in the sample. The mucosal thickening and pneumatization were the most frequent association, occurring in $8.4 \%$ of the sample. Regarding the gender, no difference was observed between the male and female genders concerning the rate of prevalence of the sinusitis in SM. In relation to age groups, difference was found only for polyp, and individuals aged over 61 years old showed higher prevalence rate. Our study shows the importance of CTCB for implant in posterior maxilla due to the higher prevalence of sinusitis found in asymptomatic patients.

DESCRIPTORS | Cone Beam Computed Tomography; Maxillary Sinus; Diagnostic Imaging.

AUTOR CORRESPONDENTE | • Ricardo Raitz Departamento de Radiologia Dentomaxilofacial, Faculdade de Medicina e Odontologia São Leopoldo Mandic • Rua Heitor Penteado, 1832, 101A São Paulo, SP, Brasil • 05438-300 E-mail: ricardoraitz@ig.com.br

- Received Jan 20, 2019 • Accepted Feb 22, 2019

- Dol http://dx.doi.org/10.11606/issn.2357-8041.clrd.2019.155150 


\section{INTRODUÇÃO}

Os seios maxilares (SM) são os maiores dos seios paranasais, onde podemos observar anormalidades por meio de exames radiográficos. Os seios paranasais têm várias funções, como: condicionamento do ar (aquecimento e umidificação), reservatórios de ar, ventilação, auxílio no olfato, redução do peso do crânio, contribuição na ressonância vocal, isolamento do cérebro, participação da formação do crânio, defesa do organismo contra agentes microbianos, e pode também proteger o cérebro em impactos. As doenças inflamatórias dos seios paranasais são decorrentes de infecção das vias aéreas superiores que podem ser causadas por vírus, bactérias, fungos ou até mesmo doenças relacionadas ao órgão dentário. ${ }^{1}$ Ademais, detectam-se as doenças sinusais inflamatórias, tais como: espessamento de membrana, pseudocisto antral, pólipos, velamento e pneumatização (perdas ósseas). ${ }^{2}$

Uma das maiores dificuldades encontradas pelo cirurgião dentista está na impossibilidade em visualizar em terceira dimensão (3D) as imagens radiográficas. É bem conhecido que as imagens radiográficas convencionais são excelentes instrumentos para visualizar as estruturas ósseas, por isso o profissional necessita possuir conhecimento para correta avaliação do que pode ser considerado normal e das possíveis anormalidades que possam acometer os SM. Algumas estruturas anatômicas podem estar sobrepostas dificultando a sua identificação nas radiografias convencionais devido às informações obtidas nestes exames serem limitadas. ${ }^{3}$ Estas limitações ocorrem porque as imagens neste tipo de exame são apresentadas de forma bidimensional e com sobreposição dos planos de estruturas anatômicas tridimensionais. ${ }^{4}$

Além disso, a avaliação minuciosa dos SM contribui para o avanço do conhecimento da anatomia destas estruturas e de suas possíveis anormalidades, fato fundamental para o diagnóstico e definição de condutas no tratamento nas áreas da odontologia e otorrinolaringologia. ${ }^{5}$ A tomografia computadorizada de feixe cônico (TCFC) favorece o planejamento adequado dos implantes dentários e o diagnóstico das possíveis anormalidades que possam acometer os SM, fato de grande importância para o profissional, pois permite identificá-las precocemente, tratá-las e acompanhálas, preservando, assim, a saúde dos pacientes e o sucesso na colocação de implantes dentários.

Portanto, o objetivo desta pesquisa foi analisar a prevalência de imagens compatíveis com sinusopatias em TCFC de pacientes selecionados para o planejamento cirúrgico de colocação de implantes.

\section{METODOLOGIA}

Esta pesquisa foi aprovada pelo Comitê de Ética da Instituição. No presente estudo foram selecionados 226 exames de TCFC de 1.500 TCFC avaliadas do banco de dados de imagens da Universidade. Todos os exames de TCFC foram realizados no aparelho Classic i-CAT (Imaging Sciences International, Hatfield, PA, EUA). Todas as imagens tomográficas foram avaliadas e visualizadas em um computador, em formato Dicom (Digital Imaging Communications in Medicine), através do software i-CAT Vision (Imaging Sciences, Hatfield, PA, EUA) em um monitor Flex Scan S20oo de 20 polegadas (Eizo Nanao Corporation $\AA$, Hakusan, Japão). O protocolo de obtenção das imagens foi: $120 \mathrm{kV}$, $38 \mathrm{~mA}$, FOV de $13 \mathrm{~cm}$, voxel de 0,25 e tempo de escaneamento de 40 segundos.

Foram incluídos os exames tomográficos de pacientes de ambos os gêneros, edêntulos e parcialmente dentados com idades entre 17 e 78 anos. Foram excluídos pacientes menores de 17 anos, pacientes com todos os elementos dentários presentes, má formação da face, apresentando lesões, pacientes sindrômicos ou politraumatizados. Também foram excluídos exames tomográficos que não incluíam a maxila como região de interesse e exames que não apresentavam boa qualidade para diagnóstico. 
Todos os dados encontrados foram tabulados em dois momentos distintos: no primeiro, anotavase o código do paciente, gênero, idade, tipo de edentulismo, e a presença de alguma sinusopatia; e, no segundo momento, caso confirmada a presença de sinusopatias, era descrita sua localização e classificação (pseudocisto antral, pneumatização, velamento sinusal, espessamento mucoso e pólipos).

Os exames de TCFC foram analisados em consenso com três especialistas em Radiologia e Imaginologia Dentomaxilofacial, com experiência de mais de cinco anos na área.

Inicialmente, para verificar a presença de imagens compatíveis com sinusopatias, utilizou-se a reformatação panorâmica. De posse da imagem, foi observada a região de interesse por meio da movimentação do cursor sobre a imagem. Em seguida, foi analisada a região de interesse por meio dos cortes transversais, gerados a partir da curva panorâmica no exame tomográfico. Nos casos em que a imagem não estivesse visível nos cortes transversais, utilizaram-se os cortes multiplanares.

Os dados coletados foram submetidos a abordagens estatísticas descritivas para avaliar a taxa de prevalência de alterações nos SM. A fim de investigar a associação entre as alterações nos SM com o gênero e a faixa etária, foram aplicados os testes: exato de Fisher e qui-quadrado. Os cálculos estatísticos foram efetuados no programa SPSS 20 (SPSS INC., Chicago, IL, EUA), tendo sido adotado o nível de significância de $5 \%$.

\section{RESULTADOS}

Neste estudo foram avaliadas 226 TCFC, as quais pertenciam a pacientes cujas idades variaram entre 17 e 78 anos, sendo que a idade média foi de 50,7 anos. Dentre as tomografias analisadas, $81(35,8 \%)$ pertenciam a pacientes do gênero masculino, enquanto 145 (64,2\%) do gênero feminino.
Dentre as 226 tomografias, em setenta $(31,0 \%)$ não se verificaram sinusopatias, enquanto nas outras 156 (69,0\%), estas estavam presentes, podendo ser sob a forma de espessamento mucoso, pólipos, pneumatização, velamento e pseudocisto antral, isolada ou simultaneamente.

Das 156 tomografias em que se verificaram sinusopatias, 62 pertenciam a pacientes do gênero masculino (39,7\%), na faixa etária entre 31 a 78 anos (média: 50,5 anos), e 94 a pacientes do gênero feminino (60,3\%), na faixa etária entre 17 e 72 anos (média: 50,4 anos). 87,2\% dos pacientes $(\mathrm{n}=136)$ apresentavam edentulismo parcial e $12,8 \%$ eram edêntulos totais $(\mathrm{n}=20)$.

Avaliando-se especificamente cada tipo de sinusopatia, observou-se que houve 63 pacientes com espessamento mucoso; sendo, portanto, sua taxa de prevalência de 27,9\% (Tabela 1). Desses 63 casos, em 41 o espessamento da membrana sinusal ocorreu unilateralmente, revelando uma taxa de prevalência de $18,1 \%$. Em vinte tomografias, o espessamento da membrana foi bilateral, indicando uma taxa de prevalência de $8,8 \%$.

Pólipos foram observados em 6 tomografias, implicando em uma taxa de prevalência de 2,7\% (Tabela 1). Em todos os casos, os pólipos foram unilaterais.

A pneumatização dos SM foi constatada em 57 tomografias, indicando uma taxa de prevalência de 25,2\% (Tabela 1). Em 22 (9,7\%) casos, a pneumatização foi unilateral. Já as ocorrências bilaterais foram vistas em 31 tomografias, representando $13,7 \%$ dos casos.

O velamento sinusal ocorreu em $69(30,5 \%)$ dos exames tomográficos avaliados (Tabela 1). Desses, 43 apresentaram velamento unilateral, indicando uma taxa de prevalência de 19,0\%. Já o velamento bilateral foi observado em 26 exames, correspondendo a 11,5\% desses casos.

O pseudocisto antral foi verificado em 29 exames $(12,8 \%)$ (Tabela 1), Sendo 24 casos unilaterais (10,6\%) e cinco casos $(2,2 \%)$ foram bilaterais. 
Tabela 1 | Prevalência de sinusopatias em TCFC.

\begin{tabular}{l|c|c} 
Alteração nos seios maxilares & $\mathrm{n}$ & Prevalência \\
\hline $\begin{array}{l}\text { Espessamento da membrana } \\
\text { sinusal }\end{array}$ & 63 & $27,9 \%$ \\
\hline Pólipo & 6 & $2,7 \%$ \\
\hline Pneumatização & 57 & $25,2 \%$ \\
\hline Velamento & 69 & $30,5 \%$ \\
\hline Pseudocisto antral & 29 & $12,8 \%$ \\
\hline
\end{tabular}

A presença simultânea de duas ou mais sinusopatias ocorreu em 56 pacientes, ou seja, em $24,8 \%$ da amostra. Especificamente, a existência concomitante de espessamento mucoso e pneumatização foi a associação mais prevalente, tendo ocorrido em $8,4 \%$ da amostra (Gráfico 1). Em seguida, correspondendo a uma taxa de prevalência de 7,1\%, foi a coexistência de espessamento mucoso e velamento. Pneumatização associada ao velamento apresentou uma taxa de prevalência de 4,4\%. Esta proporção também foi encontrada para a presença de velamento coexistente com pseudocisto antral. Menores taxas de prevalência foram verificadas para a associação entre espessamento mucoso e pseudocisto antral (3,5\%), entre pneumatização e pseudocisto antral $(3,1 \%)$ e entre espessamento mucoso, velamento e pseudocisto antral (2,2\%). As demais associações apresentaram prevalência inferior a 2,0\% ou foram inexistentes.

A Tabela 2 indica as frequências absolutas e relativas de sinusopatias de acordo com o gênero e apresenta os resultados dos testes exatos de Fisher, os quais revelaram que não houve diferença entre os gêneros masculino e feminino quanto à taxa de prevalência das alterações nos seios maxilares.

As frequências absolutas e relativas de sinusopatias de acordo com a faixa etária e os resultados dos testes de qui-quadrado encontram-se na Tabela 3. Observou-se que apenas para o pólipo a taxa de prevalência foi significativamente associada à faixa etária, sendo que indivíduos com idade acima de 61 anos apresentaram maior taxa de prevalência.

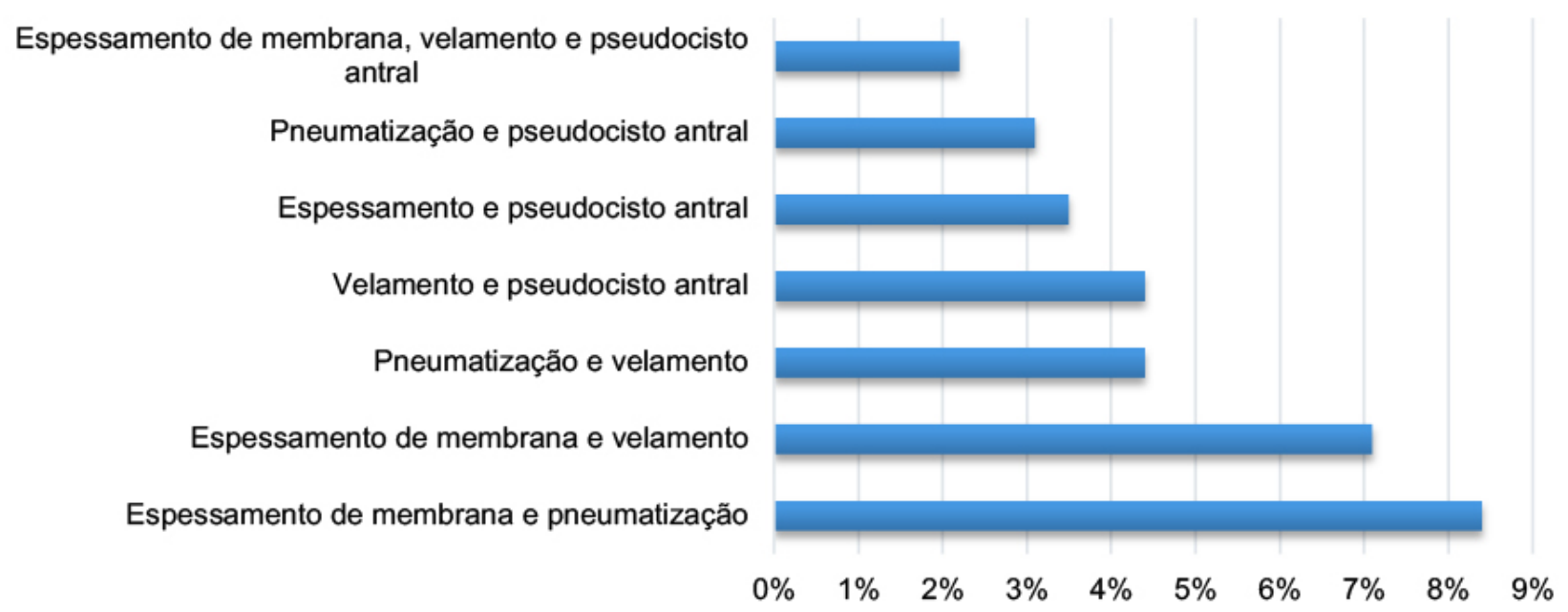

Gráfico 1 | Prevalência de duas ou mais sinusopatias em TCFC. 
Tabela 2 || Prevalência de sinusopatias em TCFC, segundo o gênero.

\begin{tabular}{|c|c|c|c|c|}
\hline \multirow{2}{*}{ Gênero } & \multicolumn{2}{|c|}{ Sinusopatias de forma geral } & \multirow{2}{*}{ Total } & \multirow{2}{*}{ valor de } \\
\hline & Presente & Ausente & & \\
\hline Masculino & $62(76,5 \%)$ & $19(23,5 \%)$ & $81(35,8 \%)$ & \multirow{3}{*}{$p=0,074$} \\
\hline Feminino & $94(64,8 \%)$ & $51(35,2 \%)$ & $145(64,2 \%)$ & \\
\hline Total & $156(69,0 \%)$ & $70(40,0 \%)$ & $226(100,0 \%)$ & \\
\hline \multirow{2}{*}{ Gênero } & \multicolumn{2}{|c|}{ Espessamento membrana sinusal } & \multirow{2}{*}{ Total } & \multirow{2}{*}{ valor de } \\
\hline & Presente & Ausente & & \\
\hline Masculino & $20(24,7 \%)$ & $61(75,3 \%)$ & $81(35,8 \%)$ & \multirow{3}{*}{$p=0,444$} \\
\hline Feminino & $43(29,7 \%)$ & $102(70,3 \%)$ & $145(64,2 \%)$ & \\
\hline Total & $63(27,9 \%)$ & $163(72,1 \%)$ & $226(100,0 \%)$ & \\
\hline \multirow{2}{*}{ Gênero } & \multicolumn{2}{|c|}{ Pólipo } & \multirow{2}{*}{ Total } & \multirow{2}{*}{ valor de $\mathrm{p}$} \\
\hline & Presente & Ausente & & \\
\hline Masculino & $3(3,7 \%)$ & $78(96,3 \%)$ & $81(35,8 \%)$ & \multirow{3}{*}{$p=0,669$} \\
\hline Feminino & $3(2,1 \%)$ & $142(97,9 \%)$ & $145(64,2 \%)$ & \\
\hline Total & $6(2,7 \%)$ & $220(97,3 \%)$ & $226(100,0 \%)$ & \\
\hline \multirow{2}{*}{ Gênero } & \multicolumn{2}{|c|}{ Pneumatização } & \multirow{2}{*}{ Total } & \multirow{2}{*}{ valor de $p$} \\
\hline & Presente & Ausente & & \\
\hline Masculino & $19(23,5 \%)$ & $62(76,5 \%)$ & $81(35,8 \%)$ & \multirow{3}{*}{$p=0,750$} \\
\hline Feminino & $38(26,2 \%)$ & $107(73,8 \%)$ & $145(64,2 \%)$ & \\
\hline Total & $57(25,2 \%)$ & $169(74,8 \%)$ & $226(100,0 \%)$ & \\
\hline \multirow{2}{*}{ Gênero } & \multicolumn{2}{|c|}{ Velamento } & \multirow{2}{*}{ Total } & \multirow{2}{*}{ valor de $p$} \\
\hline & Presente & Ausente & & \\
\hline Masculino & $31(38,3 \%)$ & $50(61,7 \%)$ & $81(35,8 \%)$ & \multirow{3}{*}{$p=0,071$} \\
\hline Feminino & $38(26,2 \%)$ & $107(73,8 \%)$ & $145(64,2 \%)$ & \\
\hline Total & 69 (30,5\%) & $157(69,5 \%)$ & $226(100,0 \%)$ & \\
\hline \multirow{2}{*}{ Gênero } & \multicolumn{2}{|c|}{ Pseudocisto antral } & \multirow{2}{*}{ Total } & \multirow{2}{*}{ valor de $p$} \\
\hline & Presente & Ausente & & \\
\hline Masculino & $14(17,3 \%)$ & $67(82,7 \%)$ & $81(35,8 \%)$ & \multirow{3}{*}{$p=0,150$} \\
\hline Feminino & $15(10,3 \%)$ & $130(89,7 \%)$ & $145(64,2 \%)$ & \\
\hline Total & $29(12,8 \%)$ & $197(87,2 \%)$ & $226(100,0 \%)$ & \\
\hline
\end{tabular}


Tabela 3 | Prevalência de sinusopatias em TCFC, segundo a faixa etária.

\begin{tabular}{|c|c|c|c|c|}
\hline \multirow{2}{*}{ Faixa etária } & \multicolumn{2}{|c|}{ Sinusopatias de forma geral } & \multirow{2}{*}{ Total } & \multirow{2}{*}{ valor de $p$} \\
\hline & Presente & Ausente & & \\
\hline Até 40 anos & $33(71,7 \%)$ & $13(28,3 \%)$ & $46(20,4 \%)$ & \multirow{4}{*}{$p=0,836$} \\
\hline $41-60$ anos & $94(69,1 \%)$ & $42(30,9 \%)$ & $136(60,2 \%)$ & \\
\hline Acima 61 anos & $29(65,9 \%)$ & $15(34,1 \%)$ & $44(19,4 \%)$ & \\
\hline Total & $156(69,0 \%)$ & $70(40,0 \%)$ & $226(100,0 \%)$ & \\
\hline \multirow{2}{*}{ Faixa etária } & \multicolumn{2}{|c|}{ Espessamento membrana sinusal } & \multirow{2}{*}{ Total } & \multirow{2}{*}{ valor de $p$} \\
\hline & Presente & Ausente & & \\
\hline Até 40 anos & $14(30,4 \%)$ & $32(69,6 \%)$ & $46(20,4 \%)$ & \multirow{4}{*}{$p=0,469$} \\
\hline $41-60$ anos & $40(29,4 \%)$ & $96(70,6 \%)$ & $136(60,2 \%)$ & \\
\hline Acima 61 anos & $9(20,5 \%)$ & $35(79,5 \%)$ & $44(19,4 \%)$ & \\
\hline Total & $63(27,9 \%)$ & $163(72,1 \%)$ & $226(100,0 \%)$ & \\
\hline \multirow{2}{*}{ Faixa etária } & \multicolumn{2}{|c|}{ Pólipo } & \multirow{2}{*}{ Total } & \multirow{2}{*}{ valor de $p$} \\
\hline & Presente & Ausente & & \\
\hline Até 40 anos & $1(2,2 \%)$ & $45(97,8 \%)$ & $46(20,4 \%)$ & \multirow{4}{*}{$p=0,028 *$} \\
\hline $41-60$ anos & $1(0,7 \%)$ & $135(99,3 \%)$ & $136(60,2 \%)$ & \\
\hline Acima 61 anos & $4(9,1 \%)$ & $40(90,9 \%)$ & $44(19,4 \%)$ & \\
\hline Total & $6(2,7 \%)$ & $220(97,3 \%)$ & $226(100,0 \%)$ & \\
\hline \multirow{2}{*}{ Faixa etária } & \multicolumn{2}{|c|}{ Pneumatização } & \multirow{2}{*}{ Total } & \multirow{2}{*}{ valor de $p$} \\
\hline & Presente & Ausente & & \\
\hline Até 40 anos & $16(34,8 \%)$ & $30(65,2 \%)$ & $46(20,4 \%)$ & \multirow{4}{*}{$p=0,227$} \\
\hline $41-60$ anos & $32(23,5 \%)$ & $104(76,5 \%)$ & $136(60,2 \%)$ & \\
\hline Acima 61 anos & $9(20,5 \%)$ & $35(79,5 \%)$ & $44(19,4 \%)$ & \\
\hline Total & $57(25,2 \%)$ & $169(74,8 \%)$ & $226(100,0 \%)$ & \\
\hline \multirow{2}{*}{ Faixa etária } & \multicolumn{2}{|c|}{ Velamento } & \multirow{2}{*}{ Total } & \multirow{2}{*}{ valor de $p$} \\
\hline & Presente & Ausente & & \\
\hline Até 40 anos & $12(26,1 \%)$ & $34(73,9 \%)$ & $46(20,4 \%)$ & \multirow{4}{*}{$p=0,705$} \\
\hline $41-60$ anos & $42(23,9 \%)$ & $94(69,1 \%)$ & $136(60,2 \%)$ & \\
\hline Acima 61 anos & $15(34,1 \%)$ & $29(65,9 \%)$ & $44(19,4 \%)$ & \\
\hline Total & $69(30,5 \%)$ & $157(69,5 \%)$ & $226(100,0 \%)$ & \\
\hline \multirow{2}{*}{ Faixa etária } & \multicolumn{2}{|c|}{ Pseudocisto antral } & \multirow{2}{*}{ Total } & \multirow{2}{*}{ valor de $p$} \\
\hline & Presente & Ausente & & \\
\hline Até 40 anos & $7(15,2 \%)$ & $39(84,8 \%)$ & $46(20,4 \%)$ & \\
\hline $41-60$ anos & $13(9,6 \%)$ & $123(90,4 \%)$ & $136(60,2 \%)$ & \\
\hline Acima 61 anos & $9(20,5 \%)$ & $35(79,5 \%)$ & $44(19,4 \%)$ & \\
\hline Total & $29(12,8 \%)$ & $197(87,2 \%)$ & $226(100,0 \%)$ & \\
\hline
\end{tabular}

* Estatisticamente significante. 


\section{DISCUSSÃO}

Embora as rinossinusites sejam muito comuns, estudos sobre a frequência e a distribuição são escassos no Brasil. ${ }^{6}$ Neste estudo, não observamos diferença estatisticamente significante entre faixas etárias para velamento, espessamento mucoso, pneumatização, pseudocisto antral, exceto para pólipo ( $\mathrm{p}=\mathrm{o}, 028)$. A taxa de prevalência foi significativamente associada à faixa etária, sendo que indivíduos com idade acima de 61 anos apresentaram maior taxa de prevalência.

De acordo com o gênero, não observamos diferença quanto à taxa de prevalência das sinusopatias nos SM. Entretanto, alguns estudos mostraram haver maior predileção dessas sinusopatias por homens. ${ }^{7-9}$

O velamento sinusal predominante entre as sinusopatias encontradas em nosso estudo mostra que os pacientes se encontram na maioria das vezes na fase inicial da rinossinusite e que devemos encaminhá-los para avaliação médica, pois o velamento sinusal pode desencadear outras doenças respiratórias quando não tratado, podendo estar também relacionado a sinusopatias de origem odontogênica..$^{10}$ É muito importante que o cirurgião dentista encaminhe o paciente para avaliação médica antes da colocação de implantes dentários para determinar as causas e origens das sinusopatias, pois a perfuração da membrana sinusal durante o procedimento cirúrgico aumenta o risco de insucesso para colocação de implantes dentários. ${ }^{5}$

Quando observamos a associação simultânea de duas ou mais sinusopatias, encontramos espessamento mucoso e pneumatização como sendo a associação mais prevalente, tendo ocorrido em $(8,4 \%)$ da amostra. O processo de pneumatização dos SM pode facilitar a disseminação de algumas infecções, fazendo com que doenças sinusais possam simular alterações odontogênicas. ${ }^{1} \mathrm{Da}$ mesma maneira, alterações odontogênicas podem se estender para os SM, simulando uma doença sinusal.
Isso pode explicar porque encontramos em nossos estudos o espessamento mucoso e pneumatização como sendo uma associação bastante prevalente. A proximidade das raízes dos dentes com a falta de osso cortical no assoalho do seio maxilar pode facilitar o aparecimento de sinusopatias. ${ }^{1}$

A TCFC desempenha um papel importante na detecção de anormalidades maxilomandibulares, em virtude do encaminhamento do paciente por várias especialidades odontológicas, além de proporcionar uma qualidade de imagem adequada para exames de tecido ósseo e reproduzir com fidelidade estruturas anatômicas, utilizando doses de radiação consideravelmente menores para o paciente do que os protocolos de tomografias computadorizadas espirais. ${ }^{8,11-19}$

Nosso estudo reforça a importância do cirurgião dentista possuir conhecimento correto da localização das estruturas anatômicas e possíveis anormalidades nos SM, pois danos nas estruturas relacionadas aos SM são graves, e as infecções podem se propagar local ou sistemicamente, o que torna de valor inestimável a avaliação do exame tomográfico da maxila. ${ }^{15}$

\section{CONCLUSÃO}

Nosso estudo mostra a importância da TCFC para implantes na região posterior da maxila em razão da alta prevalência de sinusopatias encontradas em pacientes assintomáticos, sendo que não houve diferenças em relação a gênero e idade, com exceção do pólipo.

\section{REFERÊNCIAS}

1. Bell GW, Joshi BB, Macleod RI. Maxillary sinus disease: diagnosis and treatment. Br Dent J. 2011;210(3):113-8.

2. Cavalcanti MG. Implantodontia. In: Cavalcanti MG, Marques AP, Oliva A, Perrella A, Moreira CR, Coscarelli CT, et al. Diagnóstico por imagem da face: implantodontia. São Paulo: Santos; 2008. p. 141-86.

3. Junqueira RB, Verner FS, Vilela EM, Devito KL, Chaves MGAFM, Carmo AMR. Tomografia computadorizada de feixe cônico como instrumento complementar de diagnóstico e 
planejamento cirúrgico de cisto radicular: relato de um caso clínico. Rev Odontol UNESP. 2011;40(6):338-43.

4. Lennon S, Patel S, Foschi F, Wilson R, Davies J, Mannocci F. Diagnostic accuracy of limited-volume cone-beam computed tomography in the detection of periapical bone loss: $360^{\circ}$ scans versus $180^{\circ}$ scans. Int Endod J. 2011;44:1118-27.

5. Castro AJ, Sassone LM, Amaral G. Alterações no seio maxilar e sua relação com problemas de origem odontológica. Revista HUPE. 2013;12(1):231-6.

6. Sousa CA, César CLG, Barros MBA, Carandina L, Goldbaum M, Pereira JCR. Doenças respiratórias e fatores associados: estudo de base populacional em São Paulo, 2008-2009. Rev Saúde Pública. 2012;46(1):16-25.

7. Ritter L, Lutz J, Neugebauer J, Scheer M, Dreisedler T, Zinser MJ, et al. Prevalence of pathologic findings in the maxillary sinus in cone-beam computerized tomography. Oral Surg Oral Med Oral Pathol Oral Radiol Endod. 2011;111(5):634-40.

8. Gracco A, Parenti SI, Ioele C, Bonetti GA, Stellini E. Prevalence of incidental maxillary sinus findings in Italian orthodontic patients: a retrospective cone-beam computed tomography study. Korean J Orthod. 2012;42(6):329-34.

9. Rege ICC, Sousa TO, Leles CR, Mendonça EF. Occurrence of maxillary sinus abnormalities detected by cone beam CT in asymptomatic patients. BMC Oral Health. 2012;12(30):1-7.

10. Verner FS, Junqueira RB, Visconti MAPG, Devito KL, Santos D’addázio PS, Guimarães SMR. Diagnóstico de sinusite odontogênica por tomografia computadorizada de feixe cônico. Revista ABRO. 2014;13(2):87-94.

11. Peyneau PD, Oliveira LGT, Carneiro PMR, Manzi FR. Sinusite do seio maxilar de origem odontogênica. Dental Press Endod. 2013;3(2):80-3.

12. Hashimoto K, Kawashima S, Kameoka S, Akiyama Y, Honjoya $\mathrm{T}$, Ejima K, et al. Comparison of image validity between cone beam computed tomography for dental use and multidetector row helical computed tomography. Dentomaxilofac Radiol. 2007;36(8):46571.

13. Suomalainen A, Kiljunen T, Käser Y, Peltota J, Kortesniemi M. Dosimetry and image quality of four dental cone beam computed tomography scanners compared with multislice computed tomography scanners. Dentomaxilofac Radiol. 2014;38(6):367-78.

14. Lana JP, Carneiro PM, Machado VC, Souza PE, Manzi FR, Horta MC. Anatomic variations and lesions of the maxillary sinus detected in cone beam computed tomography for dental implants. Clin Oral Implants Res. 2012;23(12):1398-403.

15. Maciel PP, Monteiro MB, Lopes PML, Sales MAO. Clinical and tomographic correlation in maxillary sinus pathologies: an evaluation by cone beam computed tomography. Pesq Bras Odontoped Clin Integr. 2012;12(4):477-81.

16. Seth V, Kamath P, Venkatesh MJ, Prasad R. Cone beam computed tomography: third eye in diagnosis and treatment planning. Int J Orthod Milwaukee. 2012;23(2):17-22.

17. Domínguez MJS, Aguilar MG, Guerra RL, Contreras GN, Aristizábal AM. Validación de la panorámica tomográfica como herramienta diagnóstica para patología del seno maxilar. Rev Fac Odontol Univ Antioq. 2013;24(2):232-42.

18. Raghav M, Karjodkar F, Sontakke S, Sansare K. Prevalence of incidental maxillary sinus pathologies in dental patients on cone-beam computed tomographic images. Contemp Clin Dent. 2014;5(3):361-5.

19. Shiki K, Tanaka T, Kito S, Wakasugi-Sato N, Matsumoto-Takeda S, Oda M, et al. The significance of cone beam computed tomography for the visualization of anatomical variations and lesions in the maxillary sinus for patients hoping to have dental implant-supported maxillary restorations in a private dental office in Japan. Head Face Med. 2014;10(20):1-13. 